$$
\text { KFK-73 }
$$

\title{
KERNFORSCHUNGSZENTRUM
}

\author{
KARLSRUHE
}

OKTOBER 1961

KFK 73

INSTITUT FUR STRAHLENBIOLOGIE

ELEKTRONISCHER PARAMAGNETISMUS IN BAKTERIOPHAGEN

A. MULLER, G. HOTZ UND K.G. ZIMMER

KERNREAKTOR

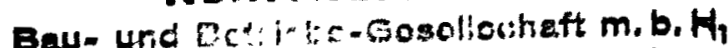
Verwakung der Zentralblicheral 


\section{Elektronischer Paramagnetismus in Bakteriophagen}

Von A. MülLer, G. Hotz und K. G. Zimmer

\section{KERNREAKTOR}

Bau- und Dos:l-se-Scoolloshaft m. b. H,

Verwaltung der Zontralbucherel 


\title{
Elektronischer Paramagnetismus in Bakteriophagen Von A. Müller, G. Hotz und K. G. Zimmer * \\ Institut für Strahlenbiologie, Kernforschungszentrum Karlsruhe \\ (Z. Naturforschg. 16 b, 658-662 [1961]; eingegangen am 25. April 1961)
}

\begin{abstract}
Purified dry preparations of coliphages TI and T2 were tested for e.s.r.-absorption in the 3-cm.band Unexpected absorption resembling very wide lines (approx. 1000 gauss) was found besides more usual absorption in viable and nonviable samples. Experimental evidence bearing on the origin of these lines is given and their meaning discussed tentatively.
\end{abstract}

Im Laufe von Untersuchungen über strahleninduzierte paramagnetische Zentren in lebenden Organismen, wie sie zum erstenmal von Zimmer, EHRENBERg und Enrenberg ${ }^{1}$ an Gerstensamen beschrieben wurden, traten einige bisher an lebenden Objekten nicht beobachtete Erscheinungen bei Phagen des Bakterienstammes $E$. coli $\mathrm{B}$ auf. Schon in den früheren Untersuchungen zeigten die untersuchten Objekte

* N. Riehl zum 60. Geburtstag in Verbundenheit und im Gedenken an zwei wechselvolle Jahrzehnte gemeinsamen Wirkens. K.G.Z. bereits unbestrahlt eine Absorption von Mikrowellen im 3-cm-Band. Diese fand sich in einem Bereich der magnetischen Feldstärke von etwa 100 Gauss Breite, in dem die Elektronen-Spin-Resonanz (e.s.r.)-Absorption von freien Radikalen gewöhnlich auftritt. An getrockneten und unbestrahlten Proben der Phagenstämme T1 und T2 fanden wir ebenfalls eine Absorption dieser Art. Bei der Untersuchung eines grö-

1 K. G. Zimmer, L. Ehrenberg u. A. Ehrenberg, Strahlentherapie 103, 3 [1957]. 
Beren Feldbereichs stellte sich jedoch heraus, daß diese relativ schmale Absorption einer sehr breiten überlagert ist $^{2}$. Die letztere erstreckt sich über mindestens einige 100 Gauss, in manchen Fällen sogar von 0 bis etwa 4000 Gauss und ist meist schon von endlicher Größe außerhalb eines magnetischen Feldes. Uber ähnliche Erscheinungen wurde bisher nur im Zusammenhang mit Untersuchungen der e.s.r.* Absorption einiger Nucleinsäuren und Nucleoproteide berichtet ${ }^{3}$.

\section{Material und Methoden}

Zur Phagenherstellung wurde als Ausgangsmaterial modifiziertes synthetisches M-9 Medium nach W e id el verwendet. Dieses enthielt pro $l 7,5 \mathrm{~g} \mathrm{Na}_{2} \mathrm{HPO}_{4} \cdot 2 \mathrm{H}_{2} \mathrm{O}$; $3,03 \mathrm{~g} \quad \mathrm{KH}_{2} \mathrm{PO}_{4} ; 1 \mathrm{~g} \mathrm{NH} \mathrm{NH}_{4} \mathrm{Cl} \quad 0,5 \mathrm{~g} \mathrm{NaCl} ; 0,2 \mathrm{~g}$ $\mathrm{MgSO}_{4} \cdot 7 \mathrm{H}_{2} \mathrm{O} ; 4,4 \mathrm{~g}$ Glucose; $0,27 \mathrm{mg} \mathrm{FeCl} \cdot 6 \mathrm{H}_{2} \mathrm{O}$. Nach dem Ansetzen mit dest. Wasser in 20-l-Flaschen gaben wir $E$. coli $\mathrm{B}$ zu und inkubierten bei $37^{\circ} \mathrm{C}$, bis eine Konzentration von $5 \cdot 10^{8}$ Bakterien $/ \mathrm{ml}$ erreicht war. Die Kultur befand sich dann noch in der exponentiellen Wachstumsphase und wurde im allgemeinen mit einem Phagen pro Bakterium infiziert und während weiterer $18 \mathrm{Stdn}$. bei $37^{\circ} \mathrm{C}$ gehalten ${ }^{4}$. Die Titration der plaquebildenden Aktivität erfolgte nach früher beschriebenen Methoden ${ }^{5}$. Das Lysat mit einem durchschnittlichen Titer von $3 \cdot 10^{11} \mathrm{Phagen} / \mathrm{ml}$ bei T2 und $2 \cdot 10^{10} \mathrm{Phagen} /$ $\mathrm{ml}$ bei TI versetzten wir mit Chloroform. Nach Abkühlung auf $4{ }^{\circ} \mathrm{C}$ fällten und reinigten wir $\mathrm{T} 2$ nach der Methode von Herriort und Barlow ${ }^{6}$ mit $\mathrm{HCl}$ bei $p_{\mathrm{H}} 4$ (Konzentration durch Zentrifugieren $45 \mathrm{~min}$ bei 12500 $\mathrm{U} / \mathrm{min})$. TI Lysate wurden mit $\left(\mathrm{NH}_{4}\right)_{2} \mathrm{SO}_{4}$ bis zur Sättigung versetzt. Nach 4 Tagen bei $4{ }^{\circ} \mathrm{C}$ waren die Phagen präzipitiert. Nach abwechselnder niedertouriger $(5000 \mathrm{U} / \mathrm{min})$ Reinigung und hochtouriger (2 Stdn. bei $15000 \mathrm{U} / \mathrm{min}$ ) Konzentration wurde die Phagensuspension in gleicher Weise wie bei T2 behandelt. Alle Präparate wurden außerdem mit RNase und DNase versetzt, restliches Coliprotein mit Antiserum ausgefällt und schließlich drei Tage gegen doppelt dest. Wasser dialysiert.

Nach der Reinigung wurden die Präparate im AcetonKohlensäure-Kältebad schnell eingefroren und anschlieBend im Vakuum gefriergetrocknet. Die dann in Form eines weißen Pulvers vorliegenden Phagen wurden mit einer Schütt-Dichte von etwa $0,5 \mathrm{~g} / \mathrm{cm}^{3}$ in die zur e.s.r.Spektrometrie verwendeten Quarzröhrchen von ca.

2 A. Müller, G. Hotz u. K. G. Zimmer, Biochem. Biophys. Res. Comm. 4, 214 [1961].

3 L. A. Blumenfeld, Biofizika (Moskva) 4, 515 [1959].

4 Die Phagenstämme (Wildtyp) und je einen Stamm E. coli B erhielten wir freundlicherweise von den Herren Prof. C. Bresch, Köln (T1) und Prof. W. Weidel, Tübingen (T2).

5 G. Horz u. A. Müller, Z. Naturforschg. 15 b, 540 [1960].

6 R. M. Herriott u. J. L. Barlow, J. gen. Physiol. 36, 17 [1952].
$3,5 \mathrm{~mm}$ Innendurchmesser gefüllt. Teilweise wurden diese Röhrchen unter Vakuum abgeschmolzen oder bei der Messung unter Vorschaltung einer mit flüssigem Stickstoff gefüllten Kühlfalle an eine Hochvakuumpumpe angeschlossen. Für einen Teil der Versuche preßten wir Tabletten von $3,5 \mathrm{~mm}$ Durchmesser und bis zu $10 \mathrm{~mm}$ Länge mit einer Dichte von $1,2 \mathrm{~g} / \mathrm{cm}^{3}$ durch $\mathrm{An}$ wendung von etwa $1000 \mathrm{~kg} / \mathrm{cm}^{2}$. Die Tabletten lieferten die gleichen Ergebnisse wie das Pulver, ließen sich jedoch leichter handhaben.

Außer an ganzen Phagen führten wir auch Versuche an deren Komponenten durch. Dazu wurde aus T2. Phagen durch osmotischen Schock und anschließende Deproteinisierung ${ }^{7}$ reine Nucleinsäure und nach HerRIOTT und BarLow ${ }^{8}$ reines Protein (ghosts) gewonnen und in lyophilisierter Form untersucht.

Für die Messungen verwendeten wir ein e.s.r.-Spektrometer der Fa. Varian, das bei einer Frequenz von $9500 \mathrm{MHz}$ arbeitete, und für die Versuche bei verschiedenen Temperaturen eine käufliche Zusatzapparatur, die Untersuchungen bei Temperaturen der Probe zwischen $-196{ }^{\circ} \mathrm{C}$ und $+300^{\circ} \mathrm{C}$ ermöglicht. Auch bei diesen Versuchen arbeiteten wir teils mit offenen, teils mit während der Messung evakuierten, teils mit abgeschmolzenen Röhrchen. Mit einer selbstgebauten Zusatzeinrichtung konnten wir bei allen Temperaturen die Proben während der Messung mit UV bestrahlen. Messungen der Konzentration der Elektronen wurden mit einem Doppelhohlraum ${ }^{9}$ durchgeführt, der mit einer Modulationsfrequenz von $100 \mathrm{kHz}$ betrieben wurde. Die Modulationsspulen dieses Doppelhohlraums bestehen nur aus je einer Windung im Inneren des Hohlraums, in einer Anordnung wie sie von INGRAM ${ }^{10}$ angegeben wurde. Um die bei unseren Versuchen erwünschte große Modulationsamplitude zu erreichen, wurden die aus einer Windung bestehenden Spulen durch eine Leitung mit möglichst kleiner Induktivität gespeist. Diese stellten wir durch Aufeinanderkleben von $10 \mathrm{~mm}$ breiten Kupferfolien mit dazwischenliegender Kunststoffolie zur Isolierung her. Diese Leitung wurde durch einen vom Röhrengenerator gespeisten Ferritkerntransformator mit nur einer Sekundärwindung mit Energie versorgt. Mit dieser Anordnung ergab sich eine maximale Modulationsamplitude von 25 Gauss.

\section{Ergebnisse}

Hochgereinigte und getrocknete Proben von T1 und T2 zeigten regelmäßig breite Absorptionsspektren, die jedoch unabhängig vom Stamm qualitative

3 M. G. Sevag, D. B. Lachmann u. J. Smolens, J. biol. Chemistry 124, 425 [1938].

8 R. M. Herriot u. J. L. Barlow, J. gen. Physiol. 40, 809 [1957].

- W. Köhnlein u. A. Mülder, Z. Naturforschg. 15 b, 138 [1960].

10 D. J. E. Ingram, Free Radicals as Studied by Electron Spin Resonance, Butterworth, London 1958, p. 82. 


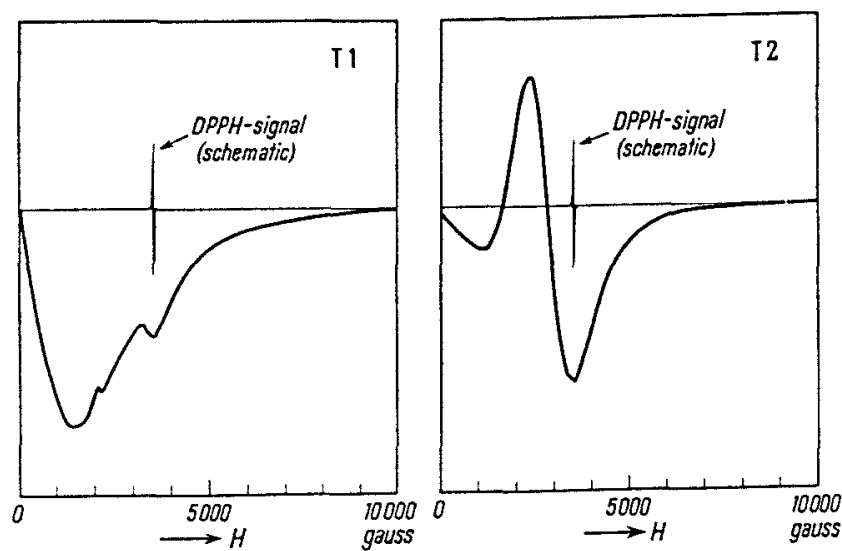

Abb. 1. Typische Beispiele der e.s.r.Absorption (erste Ableitung) von hochgereinigten Präparaten der Phagen Tl und T2. Die Messungen erfolgten bei $9,5 \mathrm{kMc}$. Zum Vergleich ist schematisch die Absorption eines freien Radikals (Diphenylpicrylhydrazyl) eingezeichnet.

Unterschiede aufwiesen, gelegentlich sogar bei verschiedenen Proben aus dem gleichen Präparat. Zwei charakteristische Spektren, bei denen die erste Ableitung der Absorption gegen die magnetische Feldstärke aufgetragen ist, sind in Abb. 1 wiedergegeben. Nach einer meist starken Abnahme von der Feldstärke Null ab, zeigte sich das erste Maximum bei etwa 1100 Gauss Feldstärke. Dieses Maximum scheint zu einer Linie zu gehören, deren Mitte im Bereich kleiner Feldstärken liegt. Dieser ersten Linie ist manchmal noch eine schwächere Linie bei 1600 Gauss überlagert, deren Breite schlecht $\mathrm{zu}$ bestimmen ist und zu $100-200$ Gauss geschätzt wird. Zwei weitere Extremwerte zeigen sich bei den Feldstärken 2300 und 3400 Gauss. Diese kann man als eine Linie bei etwa 2800 Gauss mit 1200 Gauss Breite ansehen. Bei etwa 3400 Gauss ist dann mehr oder minder groß eine relativ schmale Linie überlagert, wie sie von freien Radikalen herrührt. Bei höheren Feldstärken zeigt sich kein weiteres Maximum. Alle
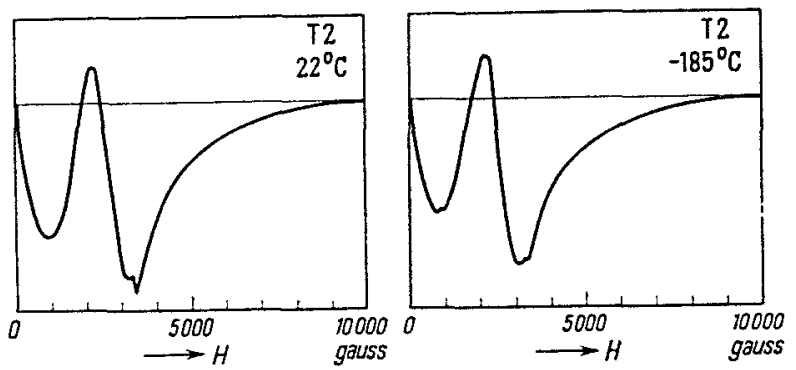

Abb. 2. Erste Ableitung der e.s.r.-Absorption eines hochgereinigten Präparats von T2 bei Zimmertemperatur und bei der Temperatur des flüssigen Stickstoffs, vorkommenden Spektren können im wesentlichen als Kombinationen dieser vier Linien aufgefaßt werden. Die Spektren hielten sich bei Aufbewahrung der Proben im evakuierten Exsikkator bei Raumtemperatur über Wochen. Bei Abkühlung der Proben auf die Temperatur flüssigen Stickstoffs zeigte sich keine Änderung (Abb. 2). Eine rasche Abnahme der Absorption verbunden mit tiefbrauner Verfärbung offener wie auch evakuiert abgeschmolzener Proben, wurde bei Erhitzung auf über $200^{\circ} \mathrm{C}$ beobachtet (Abb. 3). Eine Stde. langes Kochen von T2 vor der Gefriertrocknung veränderte das Spektrum nicht wesentlich. Bestrahlung mit ungefilterter UV-Strah. lung aus einer Hg-Niederdruckröhre gab ebenfalls keine Änderung, weder bei Raumtemperatur noch bei der Temperatur flüssigen Stickstoffs. Untersuchungen über die Einwirkung von Röntgenstrahlung
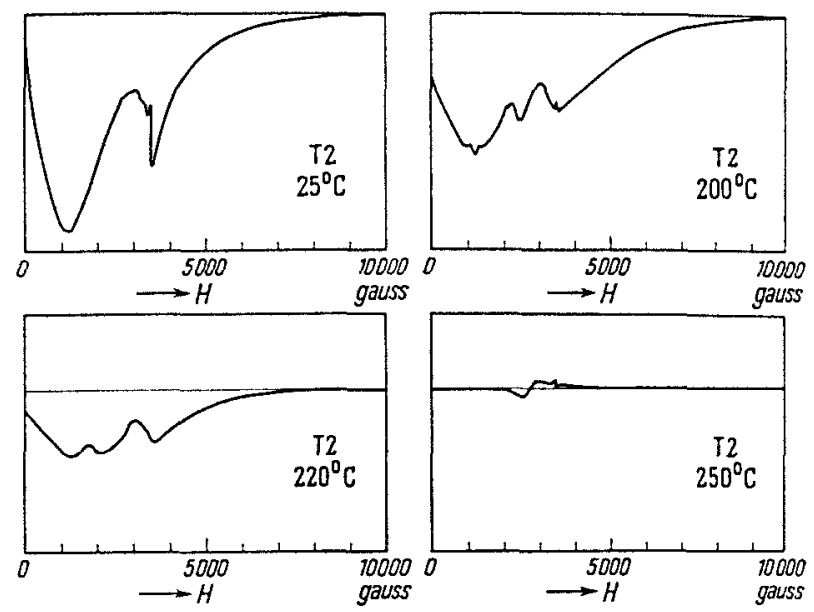

Abb. 3. Erste Ableitung der e.s.r.-Absorption eines hochgereinigten Präparats von T2 vor und nach Hitze-Behandlung.

sind noch im Gange, ihre Ergebnisse werden im einzelnen an anderer Stelle beschrieben werden. Hier sei nur vorweggenommen, $\mathrm{da}$ nur die relativ schmale Linie bei 3400 Gauss verändert wird. Bei Evakuierung der unbestrahlten Proben zeigte sich keine Änderung des Spektrums. Nach dem Einbringen in eine mit Wasserdampf gesättigte Atmosphäre verschwand das Spektrum, trat jedoch nach dem Trocknen verändert wieder auf. Eine Prüfung des zur Bakterienkultur verwendeten M-9 Mediums allein zeigte keine Absorption, weder wenn dieses Medium rein mechanisch aus den trockenen Komponenten gemischt, noch wenn es aufgelöst und gefriergetrocknet worden war. Die gereinigten und getrockneten Bakterien E. coli B allein ergaben eine 
für die Bakteriophagen charakteristische, doch nicht sehr starke Absorption. Die integrierte Absorption der Phagen zeigte beträchtliche Schwankungen. Sie war bei unkorrigierter Berechnung als paramagnetische Absorption im allgemeinen äquivalent $10^{18}$ bis $10^{19}$ Spins pro Gramm.

Die Prüfung auf Plaque-Bildung bei T2 ergab meist vollkommene Inaktivierung nach der Trocknung. Frisch getrocknete, hochgereinigte Tl-Phagen waren ohne Zusatz noch zu etwa $10 \%$ aktiv, dagegen nach Zugabe von Normalbouillon getrocknet noch zu etwa $80 \%$ aktiv. Die angegebenen Bruchteile sind als untere Grenzen zu betrachten, da sie nach Resuspension bestimmt wurden, die selbst eine Inaktivierung unbekannten Ausmaßes, besonders bei T2, herbeiführen kann. Die verschieden stark inaktivierten Phagen zeigten keine wesentlichen und reproduzierbaren Unterschiede. Elektronenoptische Bilder der getrockneten und resuspendierten T2-Phagen zeigten, daß die „Schwänze“ von den „Köpfen“ getrennt waren ${ }^{11}$.

Gereinigte Nucleinsäure aus T2 wie auch gereinigtes Hüllenprotein (ghosts) zeigten ein den ganzen Phagen ähnliches Signal. Ob dieses von den restlichen "Verunreinigungen" an Phagen oder Protein bzw. Nucleinsäure herrührt, konnte noch nicht entschieden werden.

\section{Diskussion}

Eine Diskussion der bisher durchgeführten Experimente erscheint bereits in einem frühen Stadium der Untersuchungen gerechtfertigt, da sich zwischen dem unerwarteten Auftreten starker Mikrowellenabsorption in hochgereinigten, getrockneten Bakteriophagen und den biologischen Funktionen der dafür verantwortlichen Strukturen vielleicht ein Zusammenhang zeigen läßt, der die Möglichkeit der Beobachtung solcher Funktionen an einfachen Organismen bieten würde.

Unsere ersten Bemühungen waren darauf gerichtet, denkbare Möglichkeiten für die Entstehung der beobachteten Phänomene auszuschließen, die nichts mit den biologischen Eigenschaften der untersuchten Objekte zu tun haben. Die Möglichkeit einer „mecha. nischen Verunreinigung" durch kleine Metallteile

11 Für die Herstellung der Aufnahmen sind wir den Herren Prof. Dr. W. SchäfEr und Berger, Tübingen, zu Dank verpflichtet.

12 R. G. Shulan, W. M. Walsh, H. J. Williams u. J.P. Wright, Biochem. Biophys. Res. Comm. 5, 52 [1961]. (abgerieben von den bei einem Teil der Versuche benutzten Metallwerkzeugen ${ }^{12}$ ), sei zunächst betrachtet. Abgefeilte sehr feine Späne dieser Metallteile ergaben lediglich eine Verstimmung des Probenhohlraums des Mikrowellen-Spektrometers durch unselektive, jedoch keinerlei selektive Absorption, d. h. keine Linien. Die in Metallpartikeln vorhandenen ungepaarten Elektronen können also nicht die Ursache der von uns beschriebenen Erscheinungen sein. Als nächstes kommt eine „chemische Verunreinigung " durch paramagnetische Ionen in Betracht. Diese Möglichkeit scheint uns durch die völlig andere Temperaturabhängigkeit der Spektren solcher Salze ausgeschlossen, die sich von der an Phagen -gefundenen Temperaturabhängigkeit der e.s.r.-Absorption wesentlich unterscheidet. Die Spektren paramagnetischer Ionen werden nicht schon bei $200^{\circ} \mathrm{C}$ verändert und auch nicht durch die Einwirkung von Wasserdampf. Schließlich bleibt die Möglichkeit, daß die beobachtete Absorption durch ein Zusammenwirken paramagnetischer Ionen mit dem Phagenmaterial zustandekommt ${ }^{13}$. Dies wird durch unsere Versuche nicht ausgeschlossen, würde jedoch die Bedeutung der Befunde nicht per se einschränken, weil die Rolle paramagnetischer Ionen in der molekularen Bau- und Wirkungsweise organischer Strukturen eher noch unbekannter ist als diese selbst. Aus dem gleichen Grund macht ein Beitrag des Sauerstoffs etwa in Form eines Clathrates ${ }^{14}$ die Bedeutung der Spektren nicht ohne weiteres hinfällig. Im Zusammenhang mit dieser Frage sind Versuche folgender Art interessant. Frisch präparierte, gereinigte Phagensuspensionen wurden teils mit reinem Sauerstoff, teils mit reinem Stickstoff gesättigt. Nach der anschließenden Gefriertrocknung hielten wir andere Gase von den Proben dadurch fern, daß wir alle Manipulationen in einer mit den betreffenden Gasen gefüllten gasdichten Glove-box ausführten. Ein mit T1 erhaltenes Beispiel der Spektren von so unter definiertem Gasgehalt präparierten Proben ist in Abb. 4 wiedergegeben. Die Struktur bei geringen magnetischen Feldstärken, die nach der Behandlung mit Sauerstoff stark hervortritt, ist in der unter Stickstoff präparierten Probe fast verschwunden. Die Linien bei höheren Feldstärken werden durch die Behandlung nicht beeinflußt. Wir konnten den Effekt

13 I. Isenberg, Biochem. Biophys. Res. Comm. 5, 139 [1961].

14 Auf diese Möglichkeit wurden wir von Herrn Prof. M. Delbrïck, Pasadena, hingewiesen. 


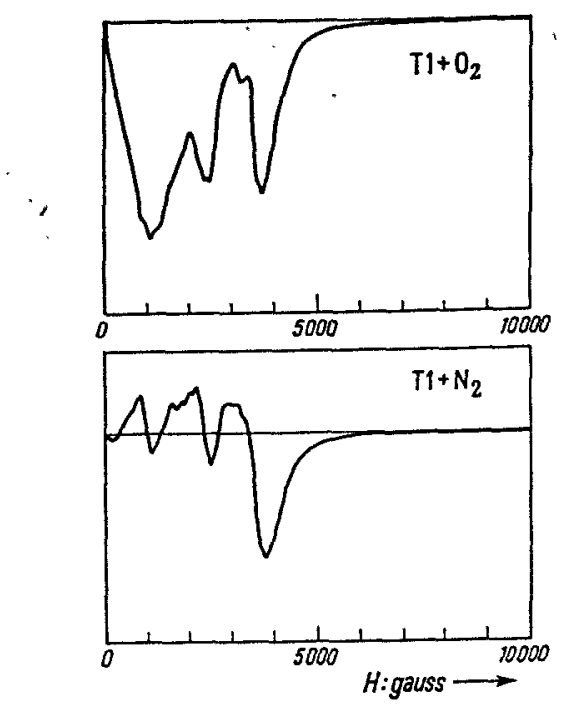

Abh. 4. Abgeleitete der e.s.r.-Absorption bei $9500 \mathrm{MHz}$ in gereinigten Phagen Tl, gesättigt mit Sauerstoff (oben) und Stickstoff (unten).

nicht beobachten, wenn der Sauerstoff nicht bereits vor der Gefriertrocknung entfernt wurde. An kommerziellen DNS-Präparaten, die auch breite Absorptionslinien aufweisen ${ }^{3}$ (jedoch nur bei höheren Feldstärken), konnte ein derartiger Sauerstoff-Effekt von uns nicht gefunden werden.

15 M. S. Blors u. J. E. Maling, Biochem. Biophys. Res. Comm. 4,252 [1961].

16 N. Rreht (N. RIL'), Migrazija energii - Novyi vid peredatschi energii $v$ mjortvoi i zhivoi materii. Gostechisdat.
Ohne auf genauere theoretische Betrachtungen eingehen zu wollen, Iassen sich nach unserer Meinung die beobachteten Erscheinungen auf einen Elektronenzustand zurückführen, bei dem durch bestimmte räumliche Anordnung von Molekülgruppen und deren periodische Wiederholung ein Zustand der energetisch niedrigste wird, der sich mit einem Triplettzustand vergleichen läßt. Durch die starke Wech. selwirkung der beiden zu solch einem Zustand gehörenden Elektronen wird die Breite des Spektrums erklärt, während das Auftreten der Linie bei 3400 Gauss auf lokale Störungen dieser Ordnung und daraus folgende Vergrößerung des Abstandes der ungepaarten Elektronen zurückgeführt werden kann. $\mathrm{Ob}$ der angedeutete Zustand nur mit oder auch ohne Beteiligung paramagnetischer Ionen oder Moleküle zustande kommen kann, wird durch unsere Versuche nicht entschieden. Weitere Versuche auch mit anderen Materialien (BLors und Maling ${ }^{15}$ ) und mit anderen Methoden, wie sie von RIEHL ${ }^{16}$ zur Untersuchung der Leitfähigkeit von Nucleinsäuren und Proteinen unternommen wurden, werden zur Klärung dieser Fragen beitragen.

Wir möchten den Herren Dr. I. Isenberg und Dr. R. G. Shulman für die Übersendung der Manuskripte ihrer Arbeiten vor der Veröffentlichung danken.

Maskva-Leningrad 1948; Naturwissenschaften 43, 145 [1956]; Ann. Physik 6. Folge 20, 93 [1957]; Kolloid-Z. 151,66 [1957]. 